\title{
Tecnura
}

ESTUDIO DE CASO

\section{Análisis de temperaturas durante la cocción de ladrillos macizos y sus propiedades finales}

\section{Analysis of temperatures during the firing bricks and final properties solid}

\author{
Gustavo Guerrero Gómez¹, Edwin Espinel Blanco², Heller Guillermo Sánchez Acevedo³
}

Fecha de recepción: 22 de marzo de 2016

Fecha de aceptación: 23 de noviembre de 2016

Cómo citar: Guerrero G., G.; Espinel B., E. y Sánchez A., H.G. (2017). Análisis de temperaturas durante la cocción de ladrillos macizos y sus propiedades finales. Revista Tecnura, 21(51), 118-131. doi: 10.14483/udistrital. jour.tecnura.2017.1.a09

\section{Resumen}

Contexto: Las propiedades finales de un bloque cerámico utilizado para mampostería dependen de las temperaturas a las que fue cocida la pasta. Dichas propiedades son relevantes, toda vez que en función de ellas será el comportamiento estructural de los muros en una edificación.

Objetivo: El propósito del presente trabajo es determinar la relación entre las propiedades finales del bloque cerámico y las temperaturas a las que fue cocido, considerando la rapidez con que estas varían en el horno.

Método: Se implementó un sistema para registrar temperaturas en el horno utilizando Labview, se consideró la velocidad de incremento de temperatura según la curva ideal para la cocción de bloques, que fueron clasificados al interior del horno, y se tomaron muestras de producto para determinar las propiedades siguiendo las normas NTC 4017 y NTC 4205.

Resultados: Se determinó la tasa de absorción inicial de agua (TIA), absorción a las 24 horas de inmersión, resistencia a la compresión y módulo de rotura (MR). Mediante un análisis de regresión lineal múltiple se encontró relación existente entre la TIA, la velocidad de incremento de temperatura y el MR. Conclusiones: Los bloques que superaron los 1000 ${ }^{\circ} \mathrm{C}$ poseen el mayor módulo de rotura (con valor de 1,54 MPa). Sin embargo, se encontró la mayor tasa de absorción inicial $\left(0,37 \mathrm{~g} / \mathrm{cm}^{2} / \mathrm{min}\right)$ y la mejor resistencia a la compresión $(7,28 \mathrm{Mpa})$ en los bloques que fueron cocidos a menor temperatura. Las propiedades no son las adecuadas dado que en el proceso de cocción no es controlada la temperatura ni el tiempo. Palabras clave: curva cocción, propiedades, ladriIlos, arcilla.

\begin{abstract}
Context: Since the estructural behavior of the walls in a building directly depends on the properties of the blocks used, these properties are very important. In particular, the final properties of a ceramic block (often used in masonry) depend on the cooking temperature of the pulp.

Objective: The purpose of this research is to determine the relationship between the final properties of a ceramic block and the temperature at which it was
\end{abstract}

1 Ingeniero mecánico, candidato a magíster en Sistemas Energéticos Avanzados. Docente e Investigador Grupo GITYD, Universidad Francisco de Paula Santander. Ocaña, Colombia. Contacto: gguerrerog@ufpso.edu.co

2 Ingeniero mecánico, especialista en Mantenimiento Industrial, magíster en Ingeniería, docente e investigador Grupo GITYD, Universidad Francisco de Paula Santander. Ocaña, Colombia. Contacto: eeespinelb@ufpso.edu.co

3 Ingeniero mecánico, doctor en Ingeniería Mecánica. Docente e investigador grupo GIEMA, Universidad Industrial de Santander. Bucaramanga, Colombia. Contacto: hgsanchez@uis.edu.co 
cooked. Additionally, it is wroth considering how quickly these temperatures vary in the kiln.

Method: In first place, a system based on Labview was used to record the temperatures in the oven. In second place, it was considered the temperature increasing rate according to the ideal curve for baking blocks, which was classified inside the oven. In third place, samples of the product were taken according to the technical norms NTC 4017 and NTC 4205 in order to determine the properties of the block.

Results: According to the samples, we determined: the Initial absorption of water (TIA), the absorption rate at 24 hours of immersion, the compressive strength, and the Modulus of Rupture (MR). Additionally, applying a multiple linear regression analysis, it was found a relationship between the TIA, the temperature increase rate, and the MR.

Conclusions: From the results in the research, it is possible to conclude that: blocks baked at more than $1000{ }^{\circ} \mathrm{C}$ have the highest modulus of rupture $(1.54$ $\mathrm{MPa})$; however, it was found that blocks baked at lower temperature presented the highest initial absorption rate $(0.37 \mathrm{gr} / \mathrm{cm} 2 / \mathrm{min})$ and the best compressive strength $(7.28 \mathrm{MPa})$. Finally, since the temperature and time are not controlled during the baking process, it is difficult for properties to be the most suitable.

Keywords: baking curve, properties, bricks, clay.

\section{INTRODUCCIÓN}

En la actualidad aún se presenta la producción de ladrillos cerámicos de forma artesanal en lo que se conoce como chircales, esto originado por la alta demanda de este producto para la construcción, el proceso fabricación comienza mezclando agua, arcilla, aserrín entre otros, hasta tener una pasta uniforme y manejable, que es llevada a un lugar abierto donde se coloca en moldes de madera; una vez retirado el molde se obtiene el ladrillo en crudo que se deja al sol por un tiempo de secado de 48 horas en promedio. Finalmente, los ladrillos son llevados a hornos de cocción artesanales durante un periodo que oscila entre 2 y 3 días a una temperatura máxima que varía entre 800 y 1300 ${ }^{\circ} \mathrm{C}$, hornos que principalmente funcionan con gas natural, carbón, y leña entre otros (Gallegos, Lang y Fernández, 2006).

Los bloques para mampostería fabricados de arcilla son utilizados en la construcción de todo tipo de edificaciones, por su disponibilidad y relación costo/beneficio debido a sus propiedades tanto estructurales como térmicas (Rozo y Sánchez, 2014), la importancia de la cerámica además de lo económico radica en que el proceso de cocción de la pasta permite la obtención de un producto duro que no es modificable en su forma, que es frágil, pero presenta resistencia mecánica y dureza, capaz de soportar agua y en algunos casos con baja conductividad térmica (Fernández, 2000).

Las propiedades mecánicas de los materiales utilizados en la construcción son consideradas para el diseño de las estructuras, en cualquier edificación los bloques de mampostería son sometidos a cargas que pueden llegar fracturarlos si no poseen las propiedades adecuadas (Valvuena, Mena y García, 2015), por lo que es indispensable para cualquier comercializador de este producto garantizar a los constructores el cumplimiento de las exigencias de acuerdo con las normas establecidas en cada país (Asociación Colombiana de Ingeniería Sísmica, 2010). Cualquier construcción está sometida a cargas combinadas y, en algunos casos, a asentamientos del terreno que ponen a prueba la estabilidad estructural de la obra, que en el caso de los muros debe ser la requerida para no aumentar la vulnerabilidad sísmica y no colocar en peligro la vida de las personas que las ocupan (Asociación Colombiana de Ingeniería Sísmica, 2004).

Sin duda, la cocción es clave, dado que en esta etapa se manifiesta si el proceso de fabricación se ha realizado correctamente y de esta depende que 
el ladrillo cocido alcance las propiedades mecánicas esperadas, según las exigencias de las normas (Aranguren, Sancho y Planas, 2011).

La cocción es un proceso fisicoquímico donde se genera movilidad atómica que une las partículas de arcilla y disminuye la porosidad; es necesario mantener la rapidez con la que se varia la temperatura en el horno, dado que un descenso súbito en la temperatura puede generar una rápida contracción y provocar tensiones que llegan a fracturar el material; igualmente se debe conocer y mantener el rango de temperatura adecuado durante la cocción, el cual depende de la pasta utilizada en cada quema (Betancurt, Martinera y Day, 2007).

\section{METODOLOGÍA}

\section{Ubicación}

El estudio se realizó en la ladrillera El Recreo, ubicada en el municipio de Ocaña, en Colombia, a una altitud de 1754 metros sobre el nivel del

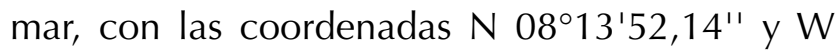
$73^{\circ} 20^{\prime} 20,39^{\prime \prime}$ con una temperatura promedio de $21^{\circ} \mathrm{C}$; el horno de sección transversal circular tiene un diámetro interior de 2,12 metros y una altura de 4,52 metros con espesor de pared de 0,24 metros, es cargado con 4300 ladrillos en cada quema.

\section{Selección de muestras}

Se organizaron los bloques en el horno por niveles; para tomar las muestras se utilizó el muestreo por estratos indicado la Tabla 1, se dividió el área del horno en 3 niveles de manera que cada uno sea claramente diferenciable con el objetivo de tomar las muestras y que estas representen el conjunto o nivel del cual provienen (Alfaro, 2002); de cada nivel se seleccionaron 20 muestras y se utilizaron cinco para cada ensayo (Instituto Colombiano de Normas Técnicas NTC 4017, 2005).

Para registrar la temperatura interna presentada en cada nivel del horno se instalaron termopares de bulbo de aleación de cromo aluminio tipo $\mathrm{K}$ con aislamiento cerámico y para las temperaturas externas termopares de alambre tipo $\mathrm{K}$ con recubrimiento fiberglass a $900^{\circ} \mathrm{F}$.

Tabla 1. Diseño del muestreo por niveles en el horno

\begin{tabular}{cll}
\hline Nivel & \multicolumn{1}{c}{ Muestra } & \multicolumn{1}{c}{ Tipo de ensayo } \\
\hline \multirow{3}{*}{1} & $1,2,3,4,5$ & Tasa de absorción inicial TIA \\
\cline { 2 - 3 } & $1,2,3,4,5$ & Ensayo de inmersión durante 24 horas \\
\cline { 2 - 3 } & $1,2,3,4,5$ & Resistencia a la compresión \\
\cline { 2 - 3 } 2 & $1,2,3,4,5$ & Módulo de rotura o flexión \\
\cline { 2 - 3 } & $6,7,8,9,10$ & Tasa de absorción inicial TIA \\
\hline \multirow{2}{*}{3} & $6,7,8,9,10$ & Ensayo de inmersión durante 24 horas \\
\hline & $6,7,8,9,10$ & Resistencia a la compresión \\
\hline & $6,7,8,9,10$ & Módulo de rotura o flexión \\
\cline { 2 - 3 } & $11,12,13,14,15$ & Tasa de absorción inicial TIA \\
\cline { 2 - 3 } & $11,12,13,14,15$ & Ensayo de inmersión durante 24 horas \\
\hline & $11,12,13,14,15$ & Resistencia a la compresión \\
\hline
\end{tabular}

Fuente: elaboración propia. 


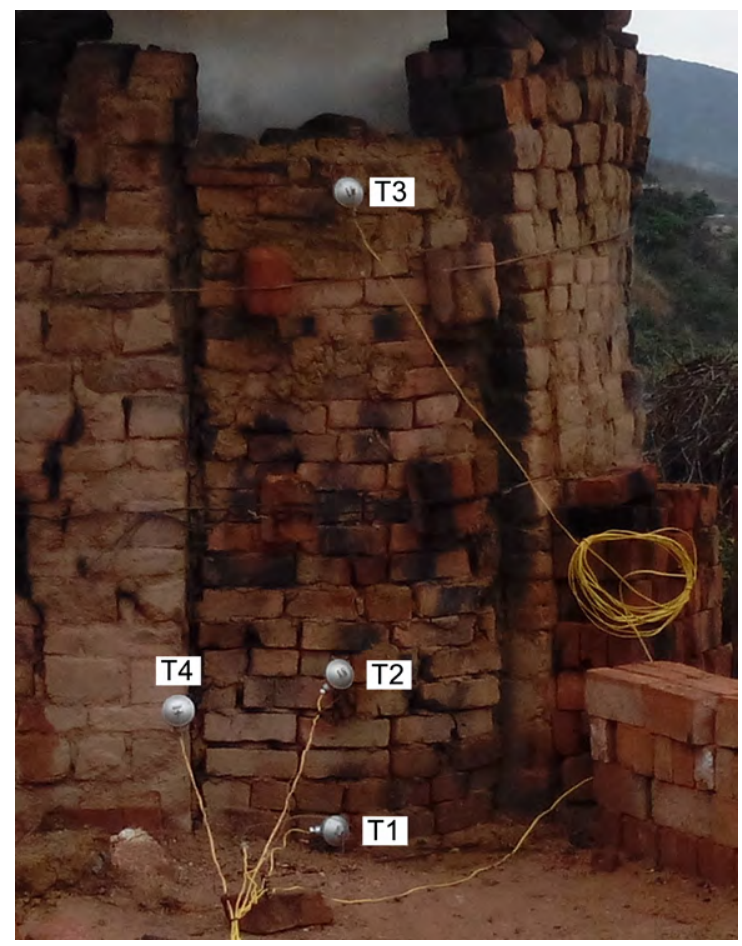

Figura 1. Termopares instalados por niveles en el horno

Fuente: elaboración propia.

\section{Diseño, programación e instalación del sistema de adquisición de temperaturas}

Se desarrolló un sistema de adquisición de datos utilizando un ejecutable en el software Labview se consideraron las variables de control y monitoreo; temperatura y tiempo de almacenamiento necesarias para la obtención de los datos, con base en las cuales se seleccionaron los comandos adecuados, de acuerdo con la programación y diseño del panel y el diagrama de bloques. Se utilizó módulo National Instruments modelo E/S NI 9213 de la serie C y un manejador DAQ Assistant del sistema embebido compact RIO, que comunica los datos con Labview. El panel de la interfaz para el usuario del sistema de adquisición permite monitorear y registrar las temperaturas en los niveles del horno estratificados durante todo el ciclo de cocción. Los registros generan tablas gráficas y numéricas, e indicadores para cada termopar. Con la librería de Labview se desarrolló el diagrama de bloques, que opera los indicadores que se colocaron en la interfaz del usuario.
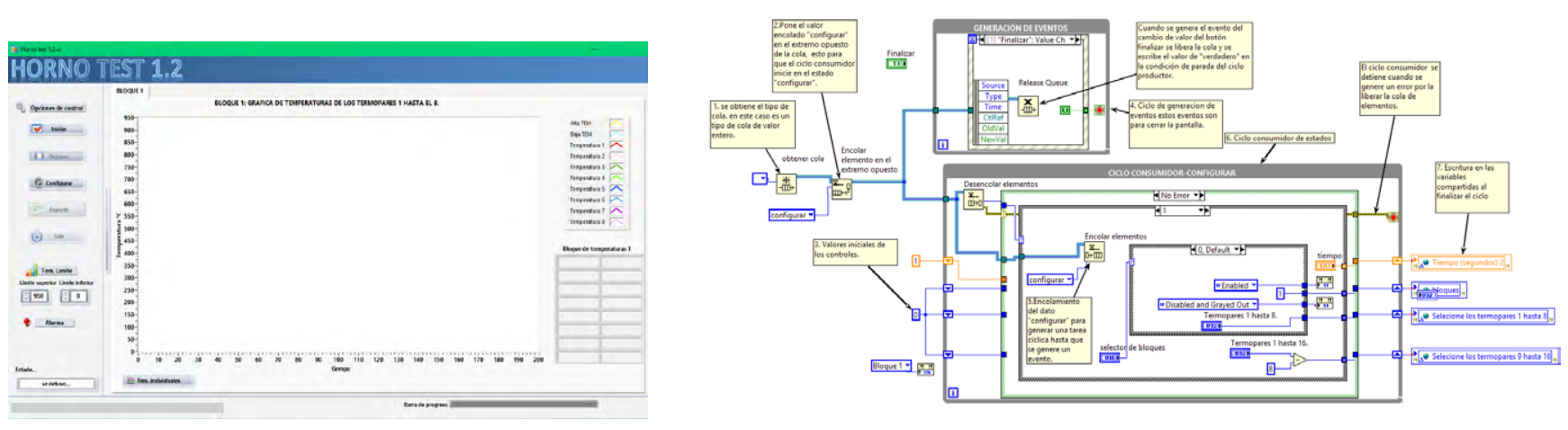

Figura 2. Panel frontal interfaz software Labview y diagrama de bloques

Fuente: elaboración propia.

\section{Tasa de absorción inicial TIA}

El ensayo se realizó de acuerdo con la norma técnica colombiana NTC 4017 a 5 muestras seleccionadas en cada nivel del horno y consistió en determinar el área de la superficie, la masa seca y la masa final; para medir las muestras se utilizó un pie de rey digital marca STANDARDGAGE con rango $350 \mathrm{~mm} \pm 0,1 \mu \mathrm{m}$, para pesarlos una balanza electrónica digital marca PINZUAR modelo PG89. 

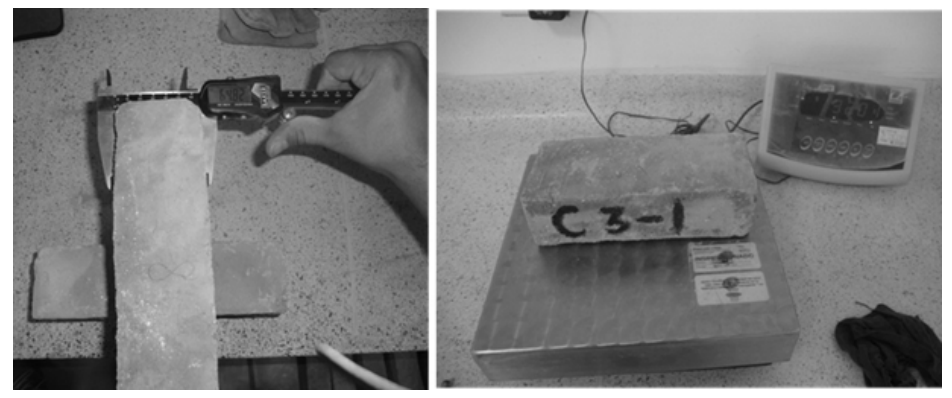

Figura 3. Determinación área de superficie y masa de las muestras

Fuente: elaboración propia.

La evaluación de la tasa de absorción inicial para cada muestra se determinó con la ecuación (1).

$$
T I A=\frac{G}{A}
$$

Donde:

$G=$ Diferencia entre la masa inicial seca y la final (g/min).

$\mathrm{A}=$ Área neta en contacto con el agua $\left(\mathrm{cm}^{2}\right)$.

\section{Ensayo de inmersión durante 24 horas}

Según la NTC 4017, se seleccionaron 5 muestras por cada nivel del horno, luego se secaron y enfriaron, se encontró su masa seca, para sumergir en agua en un rango de $15^{\circ} \mathrm{C}$ y $30^{\circ} \mathrm{C}$, por 24 horas.

La evaluación de la absorción durante las 24 horas para cada muestra se determinó con la ecuación (2).

$$
\text { \%absorción }=\left(\frac{W_{s}-W_{s s}}{W_{s}}\right) \cdot 100
$$

Donde:

$\mathrm{W}_{\mathrm{ss}}=$ masa sumergida de la muestra (g).

$\mathrm{W}_{\mathrm{s}}=$ masa seca de la muestra previa a la inmersión (g).

\section{Resistencia a la compresión}

El ensayo de compresión se realizó de acuerdo con la norma técnica colombiana NTC 4017 a las 5 muestras seleccionadas de cada nivel del horno, las cuales se secaron durante 24 horas en un horno marca Pinzuar, a temperatura entre $110{ }^{\circ} \mathrm{C}$ y $115^{\circ} \mathrm{C}$, luego se enfriaron a $24^{\circ} \mathrm{C} \pm 8{ }^{\circ} \mathrm{C}$, con valores de humedad del 30 al $70 \%$ por 4 horas, posteriormente se refrentaron con yeso aplicado en cada cara con un espesor máximo de $3 \mathrm{~mm}$, finalmente se llevaron las muestras a una universal marca Pinzuar modelo PV-100-600 serie 109 de 1000 KN, y se aplicó carga en el área de contacto hasta llevarlas a la falla.

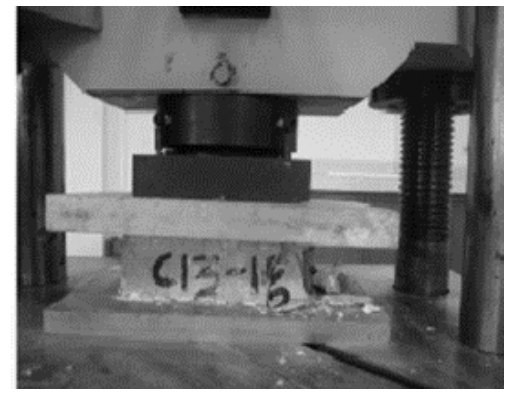

Figura 4. Aplicación de carga en la maquina universal Fuente: elaboración propia.

La resistencia a la compresión para cada muestra se determinó mediante la ecuación (3).

$$
f_{c p}^{\prime}=\frac{W}{A}
$$

Donde:

$f_{c p}^{\prime}=$ Resistencia de la muestra a la compresión (MPa). $\mathrm{W}=$ Carga máxima de rotura $(\mathrm{N})$.

$\mathrm{A}=$ Promedio de áreas brutas superior e inferior de la muestra $\left(\mathrm{mm}^{2}\right)$. 


\section{Módulo de rotura o flexión}

El ensayo se realizó de acuerdo NTC 4017, a las 5 muestras seleccionadas de cada nivel del horno, se secaron en un horno mufla marca Pinzuar modelo PG190 serie 151, por 24 horas en un rango de 110 ${ }^{\circ} \mathrm{C}$ y $115{ }^{\circ} \mathrm{C}$, de forma que se obtengan dos pesajes seguidos cada 2 horas, en los que no haya disminución superior al $0,2 \%$ de masa, una vez secadas las muestras se enfriaron a $24{ }^{\circ} \mathrm{C} \pm 8{ }^{\circ} \mathrm{C}$, entre el $30 \%$ y $70 \%$ de humedad por 4 horas, después de enfriadas se refrentaron las caras con 3,2 mm de yeso, y se aplicó carga puntual en el centro de cada cara por debajo de $1,3 \mathrm{~mm} / \mathrm{min}$ utilizando una máquina universal marca Pinzuar modelo PV100-600 serie 109 de $1000 \mathrm{KN}$.

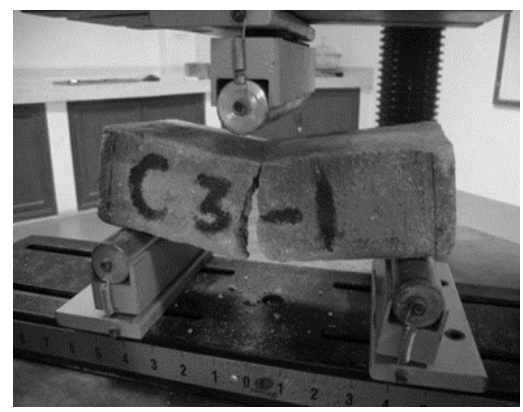

Figura 5. Ensayo de flexión

Fuente: elaboración propia.
El módulo de rotura para cada muestra se determinó mediante la ecuación (4).

$$
M R=\frac{3 W\left(\frac{L}{2}-X\right)}{B \cdot d^{2}}
$$

$\mathrm{MR}=$ Módulo rotura $(\mathrm{Pa})$.

$\mathrm{W}=$ Carga máxima de rotura $(\mathrm{N})$.

$\mathrm{L}=$ Distancia entre los soportes $(\mathrm{mm})$.

$\mathrm{B}=$ Ancho de la muestra en el plano de falla $(\mathrm{mm})$.

$\mathrm{d}=$ Profundidad de la muestra en el plano de falla $(\mathrm{mm})$.

$\mathrm{X}=$ Distancia promedio del plano de falla al centro de la pieza, medida en la dirección de la línea central de la superficie sometida a tensión $(\mathrm{mm})$.

\section{RESULTADOS}

\section{Perfiles de temperatura presentada en el horno}

Durante la quema de los ladrillos en el horno se programó el software para que registrara las temperaturas cada 5 minutos en los tres niveles en el proceso de cocción para un total de 737 registros con los cuales se realizaron las gráficas de los perfiles de temperatura.

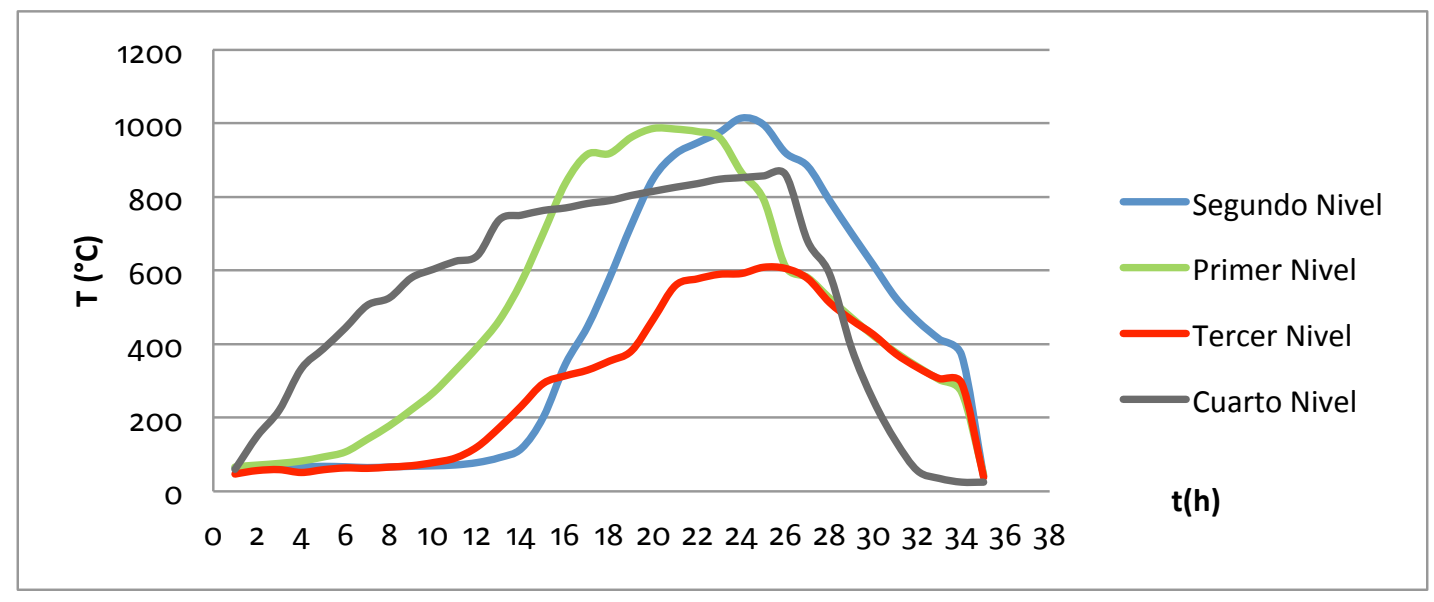

Figura 6. Curvas de temperatura en cada nivel del horno comparados con la curva ideal

Fuente: elaboración propia. 
En el nivel 1 se alcanza una temperatura máxima de $985,43{ }^{\circ} \mathrm{C}$, en el nivel 2 de $1034,53{ }^{\circ} \mathrm{C}$ y de $627,35{ }^{\circ} \mathrm{C}$ para el nivel 3 .

\section{Tasa de absorción inicial TIA}

Determina el agua que absorbe el bloque en un minuto, la cantidad de agua que absorbe un producto cerámico afecta sus propiedades finales cuando es utilizado en mampostería, lo que genera deficiencias estructurales en la edificación (Delvasto, Guzman, Torres y Cedeño, 2015).
En la tabla 2 se presentan los resultados para la absorción inicial de agua TIA de acuerdo con el procedimiento descrito en la metodología.

Se encontró que la absorción inicial de agua presenta promedios de 0,$34 ; 0,35$ y 0,37 para los niveles 1, 2 y 3 , respectivamente, y un valor promedio global de 0,35 , todos los valores están por encima de los establecidos para ladrillos en la norma NTC 4205 el cual debe ser inferior al $10 \%$.

Tabla 2. Velocidad a la que se incrementa la temperatura en cada nivel del horno

\begin{tabular}{|c|c|c|c|}
\hline & Nivel 1 (T máx. 985,43 C) & Nivel 2 (T máx. 1034,53C) & $\begin{array}{c}\text { Nivel } 3 \text { (T máx. 627,35 } \\
\left.{ }^{\circ} \mathrm{C}\right)\end{array}$ \\
\hline Rango de temperatura & Velocidad ${ }^{\circ} \mathrm{C} / \mathrm{min}$ & Velocidad ${ }^{\circ} \mathrm{C} / \mathrm{min}$ & Velocidad ${ }^{\circ} \mathrm{C} / \mathrm{min}$ \\
\hline $0^{\circ} \mathrm{C}$ hasta $400^{\circ} \mathrm{C}$ & 0,23 & 0,17 & 0,15 \\
\hline $400{ }^{\circ} \mathrm{C}$ hasta $600^{\circ} \mathrm{C}$ & 0,79 & 1,08 & 0,40 \\
\hline $600^{\circ} \mathrm{C}$ hasta $900^{\circ} \mathrm{C}$ & 1,1 & 0,92 & 0,30 \\
\hline $900{ }^{\circ} \mathrm{C}$ hasta T máxima & 0,16 & 0,42 & 0,35 \\
\hline T máxima hasta $\mathrm{T}$ amb & 0.67 & 0.92 & 0,61 \\
\hline
\end{tabular}

Fuente: elaboración propia.

Tabla 3. Resultados para la absorción inicial de agua TIA

\begin{tabular}{|c|c|c|c|c|c|c|c|}
\hline & $\underset{N^{0}}{\text { Muestra }}$ & $\begin{array}{c}\text { Área de } \\
\text { contacto } \\
\left(\mathrm{cm}^{2}\right)\end{array}$ & $\underset{(\mathrm{g})}{\text { Masa inicial }}$ & $\underset{(\mathrm{g})}{\text { Masa final }}$ & $\begin{array}{c}\text { T.I.A (g/ } \\
\left.\mathrm{cm}^{2} / \mathrm{min}\right)\end{array}$ & $\begin{array}{l}\text { T.I.A Promedio } \\
\left(\mathrm{gr} / \mathrm{cm}^{2} / \mathrm{min}\right)\end{array}$ & $\begin{array}{c}\text { Desviación } \\
\text { estándar (g/ } \\
\left.\mathrm{cm}^{2} / \mathbf{m i n}\right)\end{array}$ \\
\hline \multirow{5}{*}{$\begin{array}{l}\text { Primer } \\
\text { nivel }\end{array}$} & 1 & 284,99 & 3.413 & 3.506 & 0,33 & \multirow{5}{*}{0,34} & \multirow{5}{*}{0,10} \\
\hline & 2 & 278,31 & 3.408 & 3.496 & 0,32 & & \\
\hline & 3 & 283,66 & 3.351 & 3.423 & 0,25 & & \\
\hline & 4 & 280,08 & 3.549 & 3.628 & 0,28 & & \\
\hline & 5 & 277,12 & 3.411 & 3.555 & 0,52 & & \\
\hline \multirow{5}{*}{$\begin{array}{l}\text { Segundo } \\
\text { nivel }\end{array}$} & 6 & 270,84 & 3.077 & 3.141 & 0,24 & \multirow{5}{*}{0,35} & \multirow{5}{*}{0,12} \\
\hline & 7 & 277,98 & 3.186 & 3.269 & 0,30 & & \\
\hline & 8 & 275,61 & 3.025 & 3.131 & 0,38 & & \\
\hline & 9 & 274,94 & 2.936 & 3.017 & 0,29 & & \\
\hline & 10 & 262,61 & 2.976 & 3.117 & 0,54 & & \\
\hline \multirow{5}{*}{ Tercer nivel } & 11 & 284,61 & 3.543 & 3.650 & 0,38 & \multirow{5}{*}{0,37} & \multirow{5}{*}{0,18} \\
\hline & 12 & 282,47 & 3.364 & 3.551 & 0,66 & & \\
\hline & 13 & 284,80 & 3.427 & 3.537 & 0,39 & & \\
\hline & 14 & 285,39 & 3.587 & 3.638 & 0,18 & & \\
\hline & 15 & 290,17 & 3.586 & 3.659 & 0,25 & & \\
\hline
\end{tabular}

Fuente: elaboración propia. 


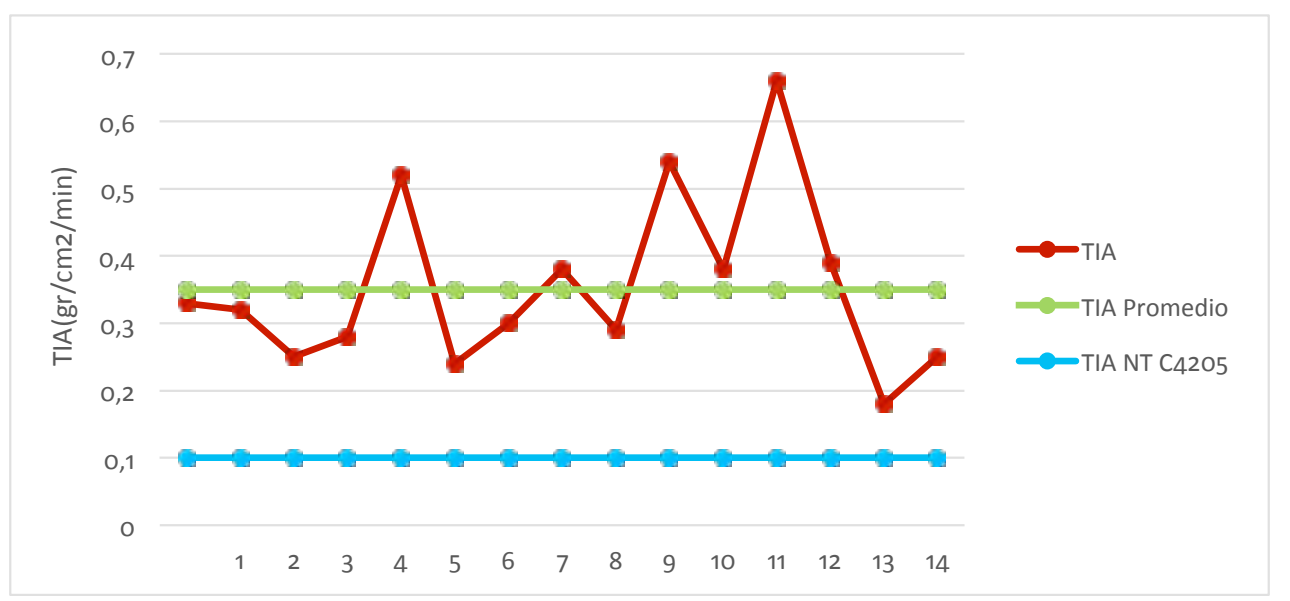

Figura 7. Tasa de absorción inicial de agua TIA

Fuente: elaboración propia.

\section{Ensayo de inmersión durante 24 horas}

La cantidad de agua que absorbe el ladrillo afecta su consistencia física y durabilidad. Se requiere conocer la capacidad del producto cerámico cuando se somete continuamente a grandes cantidades de agua o el nivel de agua que es capaz de absorber (Instituto Colombiano de Normas Técnicas NTC 4017, 2005).
La técnica usada permite determinar la absorción de agua final al término de 24 horas, las muestras ubicadas en el nivel 2 del horno son las que presentan mayor tasa de absorción con un porcentaje del $17,25 \%$, entre el nivel 1 y el nivel 3 los valores son similares $15,30 \%$ y $15,40 \%$, respectivamente.

Se consideró el porcentaje máximo promedio de absorción de agua a las 24 horas para ladrillo de mampostería no estructural interior establecido

Tabla 4. Resultados para la absorción a 24 horas

\begin{tabular}{|c|c|c|c|c|c|c|}
\hline & Muestra $N^{\circ}$ & $\begin{array}{l}\text { Masa seca } \\
\text { Ws (g) }\end{array}$ & $\begin{array}{c}\text { Masa sumergida } \\
\text { Wss (g) }\end{array}$ & $\begin{array}{c}\text { Absorción } 24 \\
\text { h (\%) }\end{array}$ & $\begin{array}{l}\text { Absorción } 24 \text { h } \\
\text { promedio (\%) }\end{array}$ & $\begin{array}{l}\text { Desviación } \\
\text { estándar (\%) }\end{array}$ \\
\hline \multirow{5}{*}{ Primer nivel } & 1 & 3.251 & 3.754 & 14,47 & \multirow{5}{*}{15,30} & \multirow{5}{*}{0,68} \\
\hline & 2 & 3.196 & 3.667 & 14,74 & & \\
\hline & 3 & 3.251 & 3.727 & 15,93 & & \\
\hline & 4 & 3.209 & 3.732 & 16,30 & & \\
\hline & 5 & 3.195 & 3.644 & 14,95 & & \\
\hline \multirow{5}{*}{$\begin{array}{l}\text { Segundo } \\
\text { nivel }\end{array}$} & 6 & 3.206 & 3.726 & 16,22 & \multirow{5}{*}{17,25} & \multirow{5}{*}{1,11} \\
\hline & 7 & 2.946 & 3.487 & 18,36 & & \\
\hline & 8 & 2.851 & 3.358 & 17,78 & & \\
\hline & 9 & 2.861 & 3.316 & 15,90 & & \\
\hline & 10 & 2.828 & 3.337 & 18,00 & & \\
\hline \multirow{5}{*}{ Tercer nivel } & 11 & 3.468 & 4.020 & 15,92 & \multirow{5}{*}{15,40} & \multirow{5}{*}{0,48} \\
\hline & 12 & 3.418 & 3.959 & 15,83 & & \\
\hline & 13 & 3.555 & 4.080 & 14,77 & & \\
\hline & 14 & 3.426 & 3.947 & 15,21 & & \\
\hline & 15 & 3.564 & 4.108 & 15,26 & & \\
\hline
\end{tabular}

Fuente: elaboración propia. 
en la norma NTC 4205 en 17,5\%, se encontraron tres valores por encima del recomendado con un valor máximo de 18,36 \% y el mínimo de 14,47 \%.

\section{Resistencia a la compresión}

Esta propiedad es importante para definir la calidad de bloque cerámico, está relacionada con la rapidez y tiempo de cocción, así como la temperatura máxima presentada en el horno.
Según la tabla 5, las muestras ubicadas en el nivel 3 presentan mayor resistencia a la compresión.

Se consideró la resistencia mínima promedio para ladrillo de mampostería no estructural establecido en la norma NTC4205 en $14 \mathrm{MPa}$, se encontró un valor promedio muy inferior de 4,87 MPa, un valor máximo de 7,91 MPa y mínimo de 2,82 MPa.

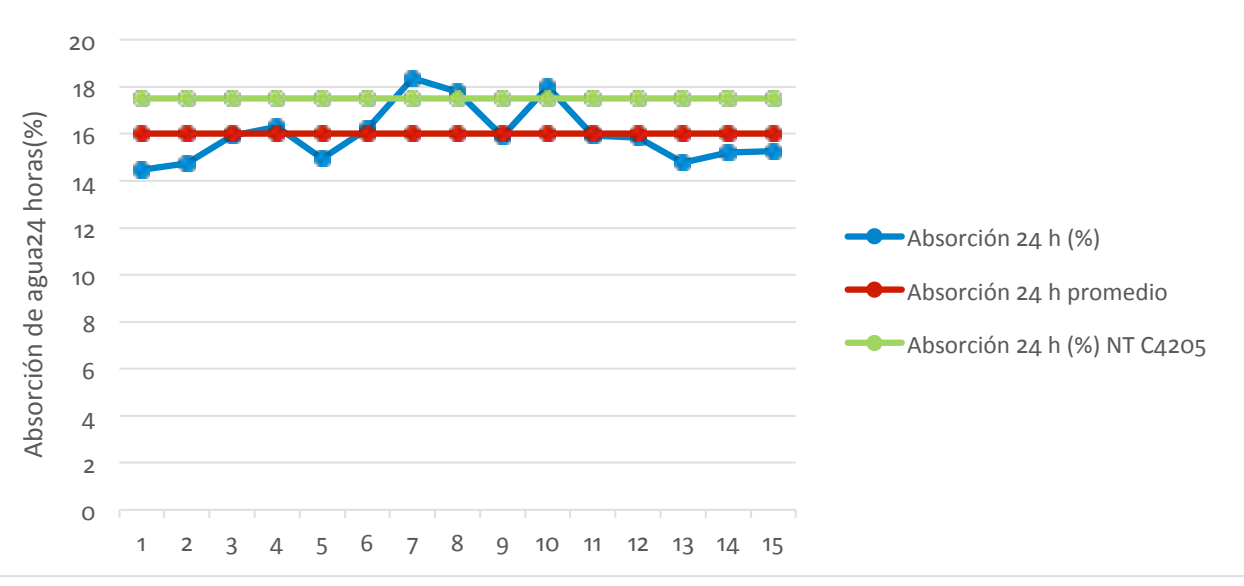

Figura 8. Absorción de agua 24 horas

Fuente: elaboración propia.

Tabla 5. Resistencia a la compresión

\begin{tabular}{|c|c|c|c|c|c|c|}
\hline & Muestra $N^{\circ}$ & $\begin{array}{c}\text { Área A } \\
\left(\mathbf{m m}^{2}\right)\end{array}$ & $\begin{array}{c}\text { Carga } \\
\text { máxima } W \\
(\mathrm{~N})\end{array}$ & $\begin{array}{c}\text { Resistencia a la } \\
\text { comprensión } \mathrm{f}^{\prime} \\
(\mathrm{Mpa})\end{array}$ & $\begin{array}{c}\text { Resistencia a la } \\
\text { comprensión } \\
\text { promedio } \mathrm{f}^{\prime}{ }_{\mathrm{cp}}(\mathrm{Mpa})\end{array}$ & $\begin{array}{l}\text { Desviación } \\
\text { estándar } \\
\text { (Mpa) }\end{array}$ \\
\hline \multirow{5}{*}{ Primer nivel } & 1 & $27.266,97$ & 77.200 & 2,83 & \multirow{5}{*}{2,93} & \multirow{5}{*}{0,10} \\
\hline & 2 & $26.872,43$ & 81.500 & 3.03 & & \\
\hline & 3 & $27.266,4$ & 82.960 & 3,04 & & \\
\hline & 4 & $27.233,04$ & 78.500 & 2,88 & & \\
\hline & 5 & $26.806,41$ & 76.850 & 2,87 & & \\
\hline \multirow{5}{*}{$\begin{array}{l}\text { Segundo } \\
\text { nivel }\end{array}$} & 6 & 23.383 & 94.340 & 4,03 & \multirow{5}{*}{4,05} & \multirow{5}{*}{1,07} \\
\hline & 7 & 26.920 & 155.330 & 5,77 & & \\
\hline & 8 & 27.025 & 102.120 & 3,78 & & \\
\hline & 9 & 28.084 & 79.080 & 2,82 & & \\
\hline & 10 & 27.116 & 104.879 & 3,87 & & \\
\hline \multirow{5}{*}{ Tercer nivel } & 11 & 28.353 & 182.640 & 6,44 & \multirow{5}{*}{7,28} & \multirow{5}{*}{0,59} \\
\hline & 12 & 28.134 & 197.610 & 7,02 & & \\
\hline & 13 & 28.238 & 218.070 & 7,72 & & \\
\hline & 14 & 28.919 & 228.850 & 7,91 & & \\
\hline & 15 & 28.188 & 201.325 & 7,14 & & \\
\hline
\end{tabular}

Fuente: elaboración propia. 


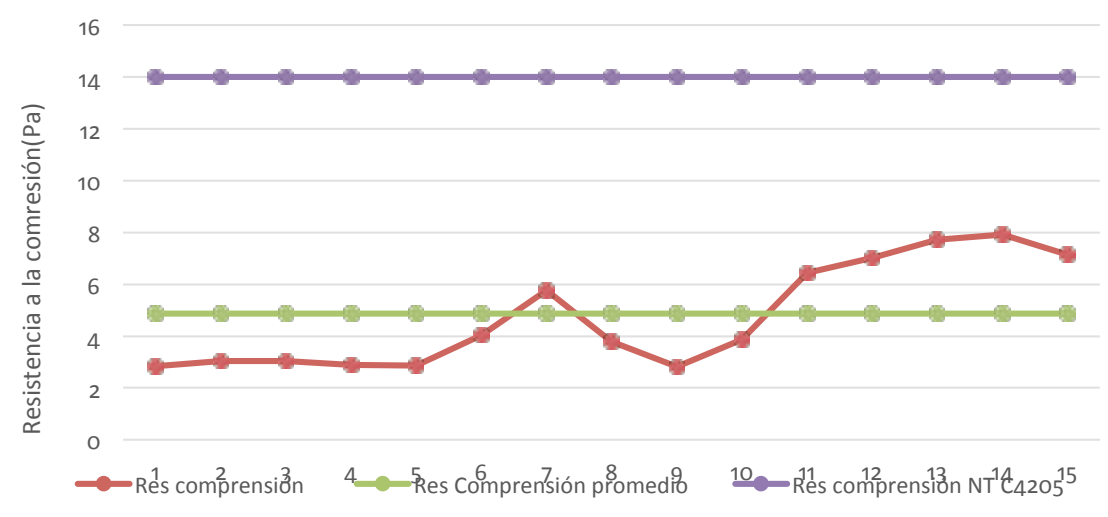

Figura 9. Resistencia a la compresión

Fuente: elaboración propia.

\section{Módulo de rotura o flexión MR.}

El módulo de rotura o ensayo de flexión (MR) permite conocer esta importante propiedad que define la durabilidad del ladrillo cuando se somete a esfuerzos de flexión, lo que ocurre frecuentemente en la mampostería (Afanador y Guerrero, 2012).

Tabla 6. Módulo de rotura o flexión

\begin{tabular}{|c|c|c|c|c|c|c|c|c|c|}
\hline & Muestra & $\begin{array}{c}\text { Carga } \\
\text { máxima } \\
\text { W (N) }\end{array}$ & $L(\mathbf{m m})$ & $B(\mathbf{m m})$ & $\underset{(\mathbf{m m})}{\mathrm{D}}$ & $\mathrm{X}(\mathrm{mm})$ & $\begin{array}{l}\text { Módulo de } \\
\text { rotura MR } \\
\quad(\mathrm{Pa})\end{array}$ & $\begin{array}{c}\text { Módulo de } \\
\text { rotura promedio } \\
\mathrm{MR}(\mathrm{Pa})\end{array}$ & $\begin{array}{c}\text { Desviación } \\
\text { estándar } \\
(\mathbf{P a})\end{array}$ \\
\hline \multirow{5}{*}{$\begin{array}{l}\text { Primer } \\
\text { nivel }\end{array}$} & 1 & $3.425,75$ & 214 & 114,34 & 75,24 & 45,33 & 0,98 & & \\
\hline & 2 & $3.124,72$ & 218 & 113,14 & 94,73 & 25 & 0,78 & & \\
\hline & 3 & $3.040,43$ & 217 & 115,42 & 76,08 & 14 & 1,29 & 1,32 & 0,51 \\
\hline & 4 & $3.016,43$ & 215 & 114,14 & 72,93 & 10,67 & 1,44 & & \\
\hline & 5 & $4.377,01$ & 213 & 113,73 & 72,38 & 11 & 2,10 & & \\
\hline \multirow{5}{*}{$\begin{array}{l}\text { Segundo } \\
\text { nivel }\end{array}$} & 6 & $1.968,75$ & 214 & 112,52 & 67,62 & 12,33 & 1,09 & \multirow{5}{*}{1,54} & \multirow{5}{*}{0,54} \\
\hline & 7 & $3.937,51$ & 215 & 110,54 & 67,56 & 8,67 & 2,31 & & \\
\hline & 8 & $2.594,9$ & 217 & 111,03 & 74,3 & 18,33 & 1,15 & & \\
\hline & 9 & $2.071,1$ & 217 & 111,76 & 65,87 & 8,33 & 1,28 & & \\
\hline & 10 & $3.786,99$ & 213 & 111,79 & 74,54 & 3 & 1,89 & & \\
\hline \multirow{5}{*}{$\begin{array}{l}\text { Tercer } \\
\text { nivel }\end{array}$} & 11 & $1.456,99$ & 241 & 115,2 & 72,4 & 30,3 & 0,65 & \multirow{5}{*}{1,45} & \multirow{5}{*}{1,38} \\
\hline & 12 & $6.454,14$ & 244 & 117,2 & 72,5 & 13,6 & 3,41 & & \\
\hline & 13 & $2.763,48$ & 242 & 116,8 & 75 & 10 & 1,40 & & \\
\hline & 14 & $1.318,52$ & 224 & 114,4 & 72 & 62,3 & 0,33 & & \\
\hline & 15 & $3.510,04$ & 224 & 114,7 & 78,6 & 16,67 & 0,13 & & \\
\hline
\end{tabular}

Fuente: elaboración propia. 


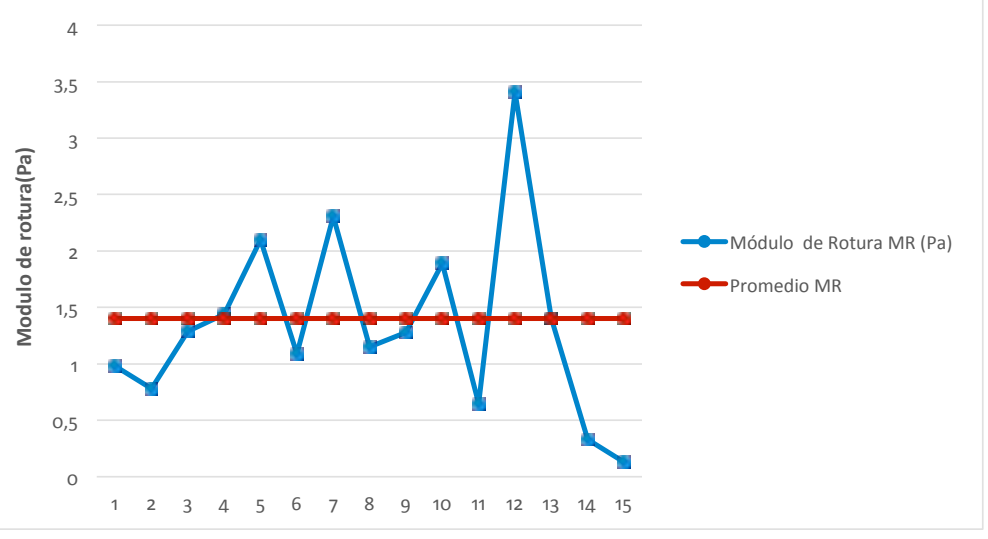

Figura 10. Módulo de rotura

Fuente: elaboración propia.

\section{Correlación entre la temperatura y las propiedades de los ladrillos}

Se realizó un análisis de correlación lineal múltiple que determina la relación funcional entre una variable dependiente $Y$ y respecto a una serie de variables independientes $X_{1}, X_{2}, X_{3}, \ldots, X_{n}$, que se expresa como la ecuación (5) (Santesmases, 2009).

$$
Y=\alpha+\beta_{1} X_{1}+\beta_{2} X_{2}+\beta_{3} X_{3}+\ldots+\beta_{n} X_{n}+c
$$

Se consideró la velocidad de incremento de temperatura, la tasa de absorción inicial TIA y los resultados de la inmersión a las 24 horas como

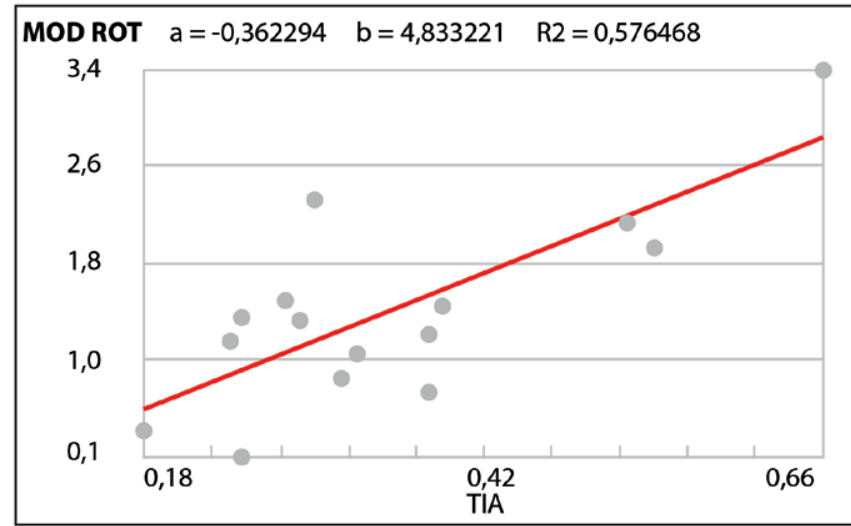

variables independientes y la resistencia a la compresión y el módulo de rotura como variables dependientes. Del análisis de la regresión lineal múltiple se encontró que la velocidad a la que se incrementa la temperatura tiene un coeficiente de correlación negativo de 0,22 con respecto a la resistencia a la compresión y positivo de 0,21 con respecto al módulo de rotura, se destaca también que la tasa de absorción al término de las 24 horas presenta un coeficiente de correlación positivo de 0,34 con el módulo de rotura. En la tabla 6, se muestra la relación encontrada entre la tasa de absorción inicial (TIA) con coeficiente de correlación positivo de 0,76 con el módulo de rotura y con un

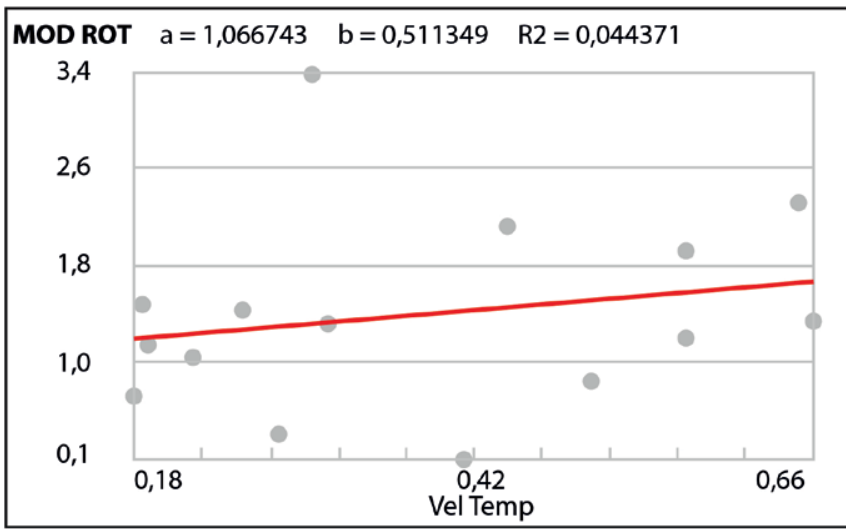

Figura 11. Regresión lineal múltiple TIA, velocidad con respecto al módulo de rotura

Fuente: elaboración propia. 
coeficiente de correlación múltiple de 0,77 para la TIA y la velocidad sobre el MR.

No se encontró relación entre la tasa de absorción inicial y la absorción a las 24 con la resistencia a la compresión como en otros trabajos (Kaushik y Rai, 2007).

\section{CONCLUSIONES}

Al comparar la curva ideal de cocción para las arcillas, con la curva real de los tres niveles en el horno, se observa un gran desfase entre ellas durante las primeras horas del precalentamiento, aunque el mayor desfase se presenta con el tercer nivel que corresponde a la parte superior del horno y es que la combustión se inicia en la parte inferior del horno y avanza en la dirección de abajo hacia arriba y la velocidad de calentamiento es lenta. Dicho desfase da lugar a una auténtica lluvia ácida sobre el material seco, ahuecando toda su estructura y manchando las superficies expuestas a los gases.

A las 6 horas de haber comenzado la cocción, la temperatura del material debería situarse sobre los $500{ }^{\circ} \mathrm{C}$ aproximadamente (Fernández, 2000); sin embargo, las mediciones efectuadas en el primer nivel no exceden los $100{ }^{\circ} \mathrm{C}$ y en los otros niveles es casi despreciable. Esto significa que se efectuó un calentamiento muy lento, precisamente en una zona en que el peligro de roturas es mínimo (García, García y Vaca, 2013).

A partir de las 6 primeras horas, se aprecia en la curva ideal una reducción gradual y progresiva de la velocidad de calentamiento (Fernández, 2000), pues la dilatación es cada vez mayor. En cambio, en el primer nivel de la curva real sucede todo lo contrario, es decir que se acelera dicha velocidad.

En la curva ideal a los $500{ }^{\circ} \mathrm{C}$ la velocidad de precalentamiento disminuye y entre los $650{ }^{\circ} \mathrm{C}$ y $750{ }^{\circ} \mathrm{C}$ se presenta un rápido aumento de la temperatura, pues la arcilla no experimenta cambios volumétricos. Luego, se presenta una uniformidad en la temperatura en toda la pieza, preparándola para entrar en la zona de máxima contracción que comienza a partir de los $750{ }^{\circ} \mathrm{C}$ (Fernández, 2000), mientras que en las curvas reales del primer y segundo nivel se presenta un rápido calentamiento que tiene lugar al acercarse la llama al material, provocando la aparición de fisuras en las caras y aristas más directamente expuestas al fuego. Además, el carbón y el material están prácticamente en contacto, con lo cual se alcanzan temperaturas muy altas, lo cual dio lugar a una vitrificación excesiva de las piezas que están más próximas a él, disminuyendo el calor y su elasticidad, lo cual dio como consecuencia una mayor sensibilidad frente a las tensiones de enfriamiento.

El enfriamiento de la curva ideal inicia entre la temperatura de régimen $800{ }^{\circ} \mathrm{C}$ y los $650{ }^{\circ} \mathrm{C}$ aproximadamente, en la que el material se encuentra en estado piroplástico y soporta sin problemas las tensiones producidas por un enfriamiento rápido (Fernández, 2000), mientras que las curvas del primer y tercer nivel en el horno están por debajo de dicha temperatura conduciendo a que el material entre en el estado elástico, soportando peor las tensiones producidas por el enfriamiento.

En el segundo nivel se presenta una temperatura de cocción que supera los $1000{ }^{\circ} \mathrm{C}$, y se encuentra para la muestras ubicadas en este nivel el mayor módulo de rotura o resistencia a la flexión con un valor de 1,54 MPa, lo que concuerda con la teoría, dado que las piezas cocidas por debajo de los $1000{ }^{\circ} \mathrm{C}$ presentan menor módulo de rotura (Peña, Miranda y Santafe, 2009). La velocidad de incremento de temperatura en el tercer nivel es la más baja y en este nivel la temperatura máxima es la menor de los tres solo alcanza los 627,35 ${ }^{\circ} \mathrm{C}$, lo que permite alcanzar la mayor tasa de absorción inicial de $0,37 \mathrm{~g} / \mathrm{cm}^{2} / \mathrm{min}$ y la mejor resistencia a la compresión de 7,28 Mpa, dado que a bajas velocidades de calentamiento se mejoran las propiedades al disminuir la temperatura máxima de cocción (Fernández, 2000).

Se encontró una incidencia de la tasa de absorción inicial (TIA) y la velocidad de incremento de temperatura en el módulo de rotura (MR), se obtuvo un coeficiente relación múltiple $r=0.77$ y un coeficiente de determinación $r^{2}=0.60$ que explica 
la proporción de la varianza del módulo de rotura explicado por el modelo, el coeficiente de regresión $\alpha=-0.53 \alpha=-0.53$, expresa el valor del MR cuando la TIA y la velocidad son iguales a 0.

Ninguna de las propiedades cumple con la norma NTC4205, en parte el proceso de cocción durante el cual no es controlada la temperatura ni el tiempo, lo que, sin duda, incide en las propiedades finales del ladrillo.

\section{FINANCIAMIENTO}

Este trabajo se desarrolló dentro del proyecto "Eficiencia energética y uso racional de la energía en la producción de materiales cerámicos en la provincia de Ocaña", el cual fue aprobado por la División de Investigación y Extensión de la Universidad Francisco de Paula Santander, Ocaña, para su ejecución se aprobó una partida de treinta y dos millones de pesos (\$32.000.000), distribuidos entre gastos de personal científico y de apoyo, equipos y materiales, servicios tecnológicos y software. La investigación realizada tiene como objetivo estudiar el proceso de cocción de material cerámico y obtención de las propiedades físico mecánicas de los ladrillos en la provincia de Ocaña, contribuyendo a la racionalización de los recursos no renovables, aumento en la productividad, beneficios económicos y la reducción de la contaminación ambiental.

\section{REFERENCIAS BIBLIOGRÁFICAS}

Afanador, N. y Guerrero, M.R. (2012). Propiedades físico-mecánicas en ladrillos macizos para mampostería. Ciencia e Ingeniería Neogranadina, 22(1), 43-58.

Alfaro, M. (2002). Introducción al muestreo minero. Santiago de Chile: Instituto de Ingenieros de Minas de Chile.

Anderson, J. (1998). Ciencia de los Materiales. 2a. ed. México: Limusa, S.A.

Aranguren, A.; Sancho, S.B. y Planas, J. (2011). Método de caracterización de las propiedades mecánicas de la fractura del ladrillo. Ciencia e Ingeniería Neogranadina, 2, 725-730.

Asociación Colombiana de Ingeniería Sísmica (2004). Guía de patologías constructivas, estructurales y no estructurales. Bogotá.

Asociación Colombiana de Ingeniería Sísmica (2010). Reglamento colombiano de construcción sismo resistente. NSR-10. Bogotá.

Betancurt, D.; Martinera, F. y Day, R. (2007). Influencia de la adición de carbonato de calcio en la eficiencia. Rev. Ing. Constr., 22(3), 187-196.

Cornellis, K.; Cornelius, K. y Hurbult, C. S. y. (1996). Manual de Minerología. 4a. ed. México: Reverte, S.A.

Dana, J.D. y Hurlbut, C.S. (1960). Manual de Mineralogía de Dana. 2a. edición. Barcelona-Buenos Aires-México: Reverté, S.A.

Delvasto, S.; Guzmán, A.; Torres, J. y Cedeño, M. (2015). Viabilidad de uso del polvo de vidrio como fundente en la elaboración de baldosas de gres porcelánico. Revista Tecnura, 19(44), 59-70.

Fernández A., M. (2000). Manual sobre fabricación de baldosas, tejas y ladrillos. España: Laboratorio Técnico Cerámico.

Gallegos, A.; Lang, B. y Fernández, M. (2006). Contaminación atmosférica por la fabricación de ladrillos y sus posibles efectos sobre la salud de los niños de zonas aledañas. Acta Nova, 3(2), 192-210.

García, C.; García, M. y Vaca, M. (2013). Resistencia mecánica de ladrillos preparados con mezclas de arcilla y lodos provenientes del tratamiento de aguas residuales. Revista Tecnura, 17(38), 68-81.

Instituto Colombiano de Normas Técnicas (2000). Ingeniería civil y arquitectura unidades de mampostería de arcilla cocida. Ladrillos y bloques cerámicos NTC 4205.

Instituto Colombiano de Normas Técnicas NTC 4017. (2005). Metodos para muestreo y ensayos de unidades de mamposteria y otros productos de arcilla. Bogotá: Icontec.

Kaushik, H. y Rai, D. (2007). Stress-strain characteristics of clay brick masonry under uniaxial compression. Journal of Materials in Civil, 19(9), 728-739. 
Peña, G.; Miranda, A. y Santafe, G. (2009). Efecto de la temperatura y tiempo de cocción en la porosidad de mezclas a base de arcillas caolines. Revista Colombiana de Física, 41(12), 27-30.

Rozo, S. y Sánchez, J.A. (2014). Propiedades físico-mecánicas de bloques H10 fabricados en el área metropolitana de Cúcuta. Ciencia e Ingeniería Neogranadina, 24(1), 67-78.

Santesmases, M. (2009). Diseño y análisis de encuesta en investigación social y de mercado. Madrid: Pirámide.
Shackelford, J. (2010). Introducción a la Ciencia de Materiales para Ingenieros. México: Pearson Educación.

Valvuena, S.; Mena, M. y García, C. (2015). Evaluación de la resistencia a la compresión en morteros de pega de acuerdo con la dosificación establecida por el código Sismo Resistente Colombiano. Estudio de caso. Revista Tecnura, 20(48), 115-122. 